\title{
SPIN POLARIZATION IN LOW-ENERGY ELECTRON DIFFRACTION: SURFACE ANALYSIS OF Pt(111)
}

\author{
Roland FEDER and Heribert PLEYER \\ Theoretische Festkörperphysik, FB 10, Universität Duisburg GH, D-4100 Duisburg, \\ Germany \\ and \\ Peter BAUER * and Norbert MÜLLER ** \\ Max-Planck-Institut für Plasmaphysik, Euratom Association, SFB 128, D-8000 München, \\ Germany
}

Received 2 February 1981

Transverse and longitudinal spin polarization components arising in the diffraction of unpolarized lowenergy electrons from $\mathrm{Pt}(111)$ have been studied as functions of energy and diffraction geometry. Experimental data measured by means of a Mott detector are in good agreement with theoretical results obtained by relativistic LEED calculations. A detailed investigation of the selective sensitivity of the calculated polarization profiles to the surface relaxation $\delta_{12}$ and to various non-structural model features firstly supports an ion-core potential involving an energy-dependent exchange approximation (by discriminating against two band structure potentials), and secondly establishes the geometry of Pt(111) as unreconstructed with a possible slight outward relaxation of the topmost atomic layer $\left(\delta_{12}=0.5 \% \pm 1.0 \%\right.$ of the bulk interlayer distance). A surface Debye temperature is found close to the bulk value $230 \mathrm{~K}$.

\section{Introduction}

In preliminary studies of spin polarization in low-energy electron diffraction (LEED) from Pt(111), experimental data measured by means of a Mott detector and corresponding theoretical results calculated by means of a relativistic dynamic LEED theory were compared $[1,2]$. The results implied a superiority of an ion-core scattering potential model with an energy-dependent exchange part over a simple band structure potential. In a more extensive theoretical and experimental investigation, on which we report in this paper, we have studied the sensitivity of calcu-

\footnotetext{
* Present address: Institut für Experimentalphysik, Universität Linz, A-4040 Linz-Auhof, Austria.

** Present address: Fachbereich Physik, Universität Osnabrück, D-4500 Osnabrück, Germany.
} 
lated polarization profiles to surface relaxation and to changes in various nonstructural model features with the aim of determining them individually by decoupling their effects.

In section 2, we outline the theoretical formalism with an emphasis on a new semi-infinite-crystal method for treating the inter-layer multiple scattering and describe specific model assumptions. An exposition of experimental aspects in section 3 is followed, in section 4, by an analysis of our results with particular focus on the effective potential model and on the surface relaxation.

\section{Theory}

\subsection{Formalism and symmetry properties}

Spin-orbit coupling and further relativistic effects are fully treated with the aid of a LEED formalism based on the Dirac equation, which has been developed earlier $[3,4]$ and is presented comprehensively in ref. [5]. We therefore give only a short outline with an emphasis on a new way of handling the inter-layer scattering problem. The formalism is of the layer-KKR type used in "intensity-only" LEED (see monographs $[6,7]$ and references therein), with the effective electron-solid interaction approximated by a complex local potential of the muffin-tin form. The solution of the scattering problem proceeds in three steps: (1) calculation of the relativistic scattering amplitudes for a single muffin-tin sphere ("crystal atom"); (2) treatment of the "intra-layer multiple scattering" in an angular momentum representation to yield the $S$-matrix and thence the transfer matrix for a single mono-atomic layer parallel to the surface, (3) combination of the individual atomic layers plus a "surface potential barrier" ("inter-layer multiple scattering") to obtain $(2 \times 2)$ scattering matrices $S_{g}$ :

$u_{g}=S_{g} u_{0}$, with $S_{g}=a_{g}+b_{g} \cdot \sigma$,

where $u_{0}$ and $u_{g}$ are the two large components of the spinor amplitudes of the incident beam and of the $g$ th diffracted beam, respectively; the vector $\sigma$ consists of the three Pauli spin matrices and $a_{g}$ and $b_{g i}(i=1,2,3)$ are complex numbers.

For the above step (3) (inter-layer scattering), previous SPLEED calculations [8] mostly assumed a finite number $M$ of layers (convergence typically requiring about 8 layers). The crystal transfer matrix was obtained as the product of the individual layer transfer matrices (actually calculated progressing in powers of two; "layer doubling"). Compared to the semi-infinite crystal Bloch wave method $[9,10]$, the finite crystal approach has the advantage of requiring significantly less computing time [11]. It involves, however, some inconvenience in computational practice: Due to exponentially increasing and decreasing factors relating to evanescent beams, the $M$-layer transfer matrix simultaneously contains - for increasing $M-$ increasingly very large and very small elements. This may lead to numerical instabil- 
ities if one includes "too many" beams. For any given energy one therefore has to optimize the number of beams $N$ and the number of layers $M$ such that convergence with respect to both $N$ and $M$ is reached without incurring an instability. In this situation, a simplification of solving the semi-infinite crystal problem, which was recently introduced by Jepsen [12] for nonrelativistic LEED calculations, constitutes an important progress. Instead of fully diagonalizing the single-layer transfer matrix $Q$, as is done in the conventional Bloch wave method (see e.g. ref. [6]), Jepsen transforms $Q$ only into a block form ("matrix splitting method"), concentrating on the eigenvalues of magnitude less than one, which correspond to the physically relevant Bloch waves carrying current into the crystal. The new method was not only found to require less computing time than the old Bloch wave method, but also anticipated to be faster than the finite-crystal "layer-doubling" method [12]. We therefore adopted it for use in our relativistic LEED programme. Computational tests on $\mathrm{Pt}(111)$ and $\mathrm{Ni}(001)$ were found to reproduce results previously obtained by "layer-doubling", with a 25-30\% time gain in computing the bulk reflection matrix from the single-layer transfer matrix. Remembering the abovementioned inconvenience in using "layer-doubling", Jepsen's semi-infinite crystal method thus appears to be clearly superior. The $(2 N \times 2 N)$ bulk reflection matrix obtained by this method is then combined in our programme with the $(4 N \times 4 N)$ transfer matrix of a "selvedge" (surface potential barrier plus a mono-atomic layer), spaced by a variable distance (topmost interlayer spacing) from the bulk, to yield the scattering matrices $S_{g}$ (cf. eq. (1)) for the entire system.

For an unpolarized primary beam, as is used in our experiment, the spin polarization vector $\boldsymbol{P}_{\boldsymbol{g}}$ of the $g$ th diffracted beam is obtained (cf. refs. [13] and [14]) from $S_{g}$ as

$\boldsymbol{P}_{g}=\left[2 \operatorname{Re}\left(a_{g} b_{g}^{*}\right)-\mathrm{i}\left(b_{g}^{*} \times b_{g}\right)\right] /\left(\left|a_{g}\right|^{2}+\left|b_{g}\right|^{2}\right)$.

Without numerical calculations, time reversal and spatial symmetries allow general predictions about $\boldsymbol{P}$ (we now drop the beam index $g$ ) [13,14], of which the following are relevant for the present study on $\operatorname{Pt(111).~(1)~If~the~scattering~plane,~defined~}$ by the wave vectors $k_{0}$ and $k$ of the incident and the $g$ th diffracted beam, coincides with a mirror plane of the crystal, $P$ is parallel to $n=\hat{k}_{0} \times \hat{k}$ (with $\hat{k}_{0}=k_{0} /\left|k_{0}\right|$, $\hat{k}=\boldsymbol{k} /|\boldsymbol{k}|$ ), i.e. there is only a transverse component $P_{n}=\boldsymbol{P} \cdot \boldsymbol{n}$ normal to the scattering plane. (2) If the mirror plane condition is not fulfilled, multiple scattering generally produces nonvanishing components in the scattering plane: a longitudinal one $P_{k}=\boldsymbol{P} \cdot \hat{k}$ and a transverse one $P_{\boldsymbol{e}}=\boldsymbol{P} \cdot \boldsymbol{e}$, where $\boldsymbol{e}=\hat{k} \times \boldsymbol{n}$. (3) If the surface normal is a three-fold rotation axis of the crystal (e.g. for $\operatorname{Pt}(111)$, which belongs to the symmetry group $C_{3 v}$ ), specular beam rotation diagrams $P(\varphi)$ exhibit only three-fold symmetry, whilst intensity $I(\varphi)$ and degree of polarization $P(\varphi)=|P(\varphi)|$ diagrams are six-fold as a consequence of time reserval invariance. (4) If there are mirror planes normal to the surface (like for $\mathrm{C}_{3 \mathrm{v}}$ ), specular beam rotation diagrams $P_{n}(\varphi)$ are symmetric, $P_{k}(\varphi)$ and $P_{e}(\varphi)$ antisymmetric with respect to these planes. 


\subsection{Model assumptions for Pt(111)}

We initially $[1,2]$ chose Pt(111), because the geometry of this surface is simple and essentially known from LEED intensity analyses $[15,16]$ and $\mathrm{MeV}$ ion scattering (ref. [17]) and references therein). These studies agree on a model consisting of a simple bulk truncation with a possible small uniform outward relaxation of the outermost atomic layer. They differ, however, somewhat with regard to the magnitude of this deviation $\delta_{12}$ of the topmost interlayer spacing from the bulk value. While ref. [16] gives $\delta_{12}=1 \% \pm 4.4 \%$, ref. [15] reports $0 \% \pm 2.5 \%$ and ref. [17] $0 \%+0.4 \%$. In the present calculations, $\delta_{12}$ was varied between $-4 \%$ and $+4 \%$ in view of independently determining its actual value by comparing calculated and measured spin polarization profiles.

In order to assess the influence of details of the single-sphere (muffin-tin) potential, in particular of the exchange approximation, on calculated spin polarization profiles, we used the following three potentials: (1) A relativistic non-self-consistent band structure potential $V_{A}[18]$ obtained by overlapping relativistic atomic charge densities [19] with exchange treated in the full Slater local density approximation [20]. (2) A relativistic self-consistent band structure potential $V_{G}[21]$ obtained from similar atomic charge densities using a local RPA exchange approximation [22]. (3) A potential $V_{E}$, which we constructed from the charge density associated with $V_{G}$ using a local exchange approximation, which decreases with increasing energy $E$ of the incident electron [23].

From these real "crystal atom" potentials, scattering phase shifts $\delta_{l}^{ \pm}$up to $l=7$ were obtained by solving the radial Dirac equation as in ref. [24]. Thermal lattice vibrations were taken into account by averaging the relativistic atomic scattering amplitudes over a Debye spectrum (at $T=300 \mathrm{~K}$ ), which implies replacing the real $\delta_{l}^{ \pm}$in our formalism by effective complex $\tilde{\delta}_{l}^{ \pm}$. Since a substantial reduction of the perpendicular-vibration surface Debye temperature has been reported for $\operatorname{Pt}(111)$ [25-28], we have performed calculations assuming Dehye temperature values ranging from the bulk value $T_{\mathrm{D}}^{\mathrm{b}}=230 \mathrm{~K}$ down to $115 \mathrm{~K}$ either for the topmost layer only (with $230 \mathrm{~K}$ for the other layers) or, in the sense of an effective Debye temperature, for all layers [29]. Anisotropy between perpendicular and parallel surface atom vibrations, which may have some effect on LEED [30], has been disregarded, since our interest concentrated, in order to determine surface relaxation and ion core potential, on polarization profiles, which turned out to be only weakly sensitive to deviations of $T_{\mathrm{D}}^{\mathrm{s}}$ from the bulk value. The further refinement of an anisotropic $T_{\mathrm{D}}^{\mathrm{s}}$ can be expected to be immaterial for these profiles.

The real part $V_{\mathbf{r}}$ of the inner potential was determined as $12 \mathrm{eV}$ by comparison of calculated and measured normal-incidence polarization versus energy profiles (cf. ref. [1]). For the imaginary part we used the standard values 3,4 and $5 \mathrm{eV}$. For the surface potential barrier we assumed, in addition to the "no-reflection" model, a real exponential form $V_{\mathrm{r}} /[1+\exp (-z / \alpha)]$, with $\alpha=0.7 \AA$, joined smoothly to the asymptotic image form, plus a Gaussian imaginary part (cf. ref. $[8])$. 


\section{Experiment}

Earlier versions of the apparatus used in the experiment have been described in refs. [31] and [32]. Here a detailed description of the modified set-up is given, which was briefly sketched in ref. [2]. The apparatus (see fig. 1) consists of two parts: the ultra-high-vacuum diffraction system, which is operated at a pressure less than $6 \times 10^{-11} \mathrm{mbar}$ and the high-vacuum Mott detector, which works at less than $10^{-7}$ mbar. In the diffraction system an unpolarized electron beam is diffracted by the (111) surface of a platinum crystal. The inelastically scattered electrons are filtered out by a retarding field in a three grid LEED optics. The elastically diffracted beam, the polarization of which is to be analyzed, is fed through apertures in the grids and a probe hole in the fluorescent screen to the polarization analyzer (i.e. the Mott detector). Using Mott scattering for the measurement of spin polarization implies a fixed direction of the diffracted beam under consideration. To allow for a variation of the electron energy and the polar and azimuthal angles of incidence for any diffracted beam, both the electron gun and the crystal must be moveable: the crystal can be rotated about two axes. One of them lies within the crystal surface plane (polar axis), the other within $0.15^{\circ}$ perpendicular to the crystal surface (azimuthal axis). The LEED electron gun may be rotated about the polar axis of the crystal about $270^{\circ}$. Thus for any chosen energy any diffracted beam may be fed to the polarization analyzer. The only limiting condition is that the scattering plane always contains the surface normal of the crystal.

After the electron beam has passed through the probe hole it is focussed and electrostatically deflected by $90^{\circ}$. Subsequently it is accelerated to $120 \mathrm{keV}$ and

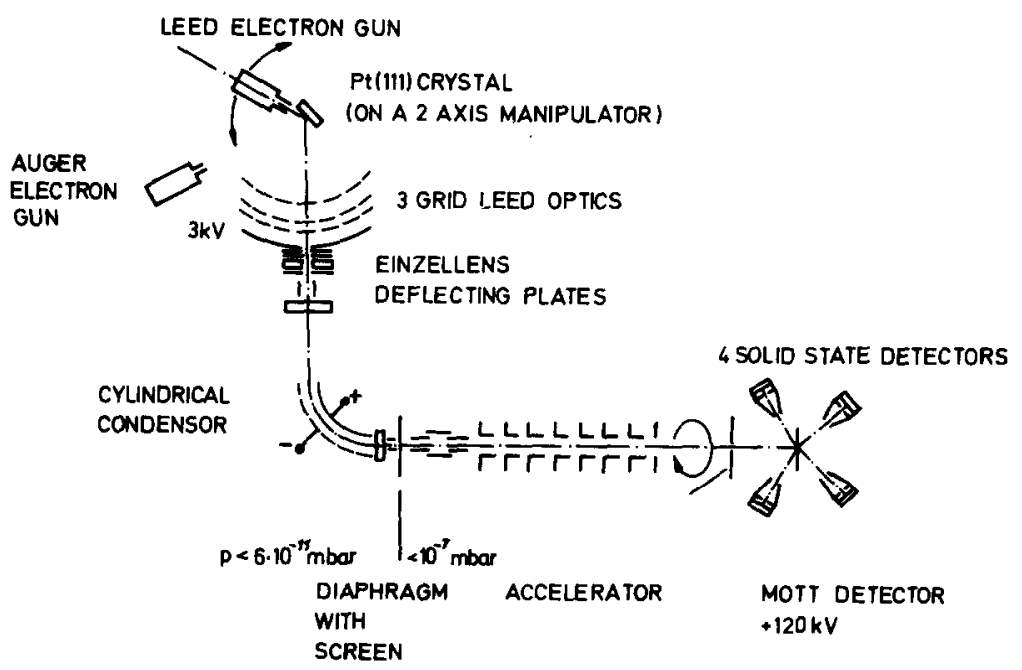

Fig. 1. Experimental set-up. 
focussed to the aperture at the entrance of the Mott scattering chamber. The $90^{\circ}$ deflection is done within the scattering plane of diffraction. Thus the projection $P_{n}$ of the polarization vector onto the normal to the scattering plane remains transverse, while the longitudinal component $P_{k}$ (parallel to the momentum $k$ of the diffracted beam) becomes transverse after the deflection. $P_{n}$ and $P_{k}$ can now be measured by orienting the scattering plane in the Mott detector perpendicular to their respective direction. In this scattering plane there are two electron counters at a scattering angle of $120^{\circ}$ which yield an intensity asymmetry proportional to $P_{n}$ and $P_{k}$, respectively. Two additional counters are used to correct for apparative asymmetries.

Special care was taken in crystal preparation and mounting as well as in optimizing the diffraction geometry. The Pt crystal was spark-cut from a single crystal rod. Its surface was oriented to the (111) crystallographic plane within $0.1^{\circ}$ by means of an X-ray diffractometer and mechanically polished with diamond paste from $50 \mu \mathrm{m}$ down to $0.25 \mu \mathrm{m}$. The crystal was mounted on a platinum-covered heater (Varian) by platinum wires and cleaned in aqua regia and alcohol. In vacuo the crystal was further cleaned by repeated heating cycles to about $1100 \mathrm{~K}$ at $10^{-6}$ to $10^{-7}$ mbar $\mathrm{O}_{2}$ for several hours alternating with argon-bombardment in the energy range of 1 to $3 \mathrm{keV}$. By this procedure, impurities (mainly carbon) contained in the crystal were removed. During the measurements the crystal was kept clean by repeated heating to $600 \mathrm{~K}$ and by bombardment with 1 to $2 \mathrm{keV}$ neon ions at grazing incidence. The chemical purity of the crystal surface was checked by Auger electron spectroscopy using a $3 \mathrm{keV}$ glancing-incidence electron gun for the excitation of the Auger electrons and the LEED system combined with a lock-in amplifier for their evaluation. The experimental data are given with error bars according to the statistical errors due to the counting in the Mott detector. In addition, there are the following systematical uncertainties: for changes in energy \pm 0.1 $\mathrm{eV}$, for absolute values of energy $\pm 1 \mathrm{eV}$, for changes in polar angle $\pm 0.1^{\circ}$, for absolute values of polar angle less than $0.5^{\circ}$, for changes of azimuthal angles $\pm 0.4^{\circ}$, for polarization zero $\pm 1 \%$ and for polarization sensitivity of the Mott detector $\pm 7 \%$ of the measured values.

\section{Surface analysis of $\operatorname{Pt}(111)$}

The normal and longitudinal components $P_{n}$ and $P_{k}$ of the spin polarization vector $\boldsymbol{P}$ of various (elastic) LEED beams were measured versus energy $E$, polar angle $\vartheta$ (defined with respect to the surface normal) and azimuthal angle $\varphi$ ("polarization profiles" $\boldsymbol{P}(E, \vartheta, \varphi)$ with two of the three variables fixed). Corresponcing numerical calculations were carried out for a variety of models characterized by different physically reasonable values of structural and non-structural quantities (cf. section 2.2). The set of values, which consistently leads to optimal agreement between the calculated and the experimental polarization profiles is regarded as "real" [33]. The 
accuracy of this "surface analysis" rests upon the sensitivity of the polarization profiles against small changes in the model quantities. For the method to be practicable it is essential to find polarization profiles, which are highly sensitive to a particular model quantity (e.g. the ion-core potential) and insensitive to the others, i.e. to decouple the effects of the different model quantities. In the following, we present and discuss our results, illustrating the determination of the ion-core potential and of the surface relaxation of $\mathrm{Pt}(111)$ by typical selectively sensitive spin polarization profiles.

Representative of specular beam rotation diagrams, fig. 2 shows experimental and "best" theoretical $P_{n}, P_{k}$ and $P_{e}$ versus azimuthal angle $\varphi$ profiles for $E=80 \mathrm{eV}$ and $\vartheta=47^{\circ}$. The $C_{3 \mathrm{v}}$ symmetry (three-fold rotation symmetry about the surface normal plus mirror planes at $\varphi=0,60^{\circ}, 120^{\circ}$ ) provides a check on the quality of the experiment (in particular regarding the alignment of the rotation axis normal to the surface). Bearing in mind that the mirror operation transforms $P_{n} \rightarrow P_{n}, P_{k} \rightarrow$ $-P_{k}$, and $P_{e} \rightarrow-P_{e}$, the experimental $P_{n}$ and $P_{k}$ data are seen to have the required symmetries. Moreover, the following predictions from general symmetry arguments $[13,14]$ are explicitly confirmed by experiment and numerical calculations: (1) for $\varphi=0,60^{\circ}$ and $120^{\circ}$, for which the scattering plane coincides with a mirror plane of

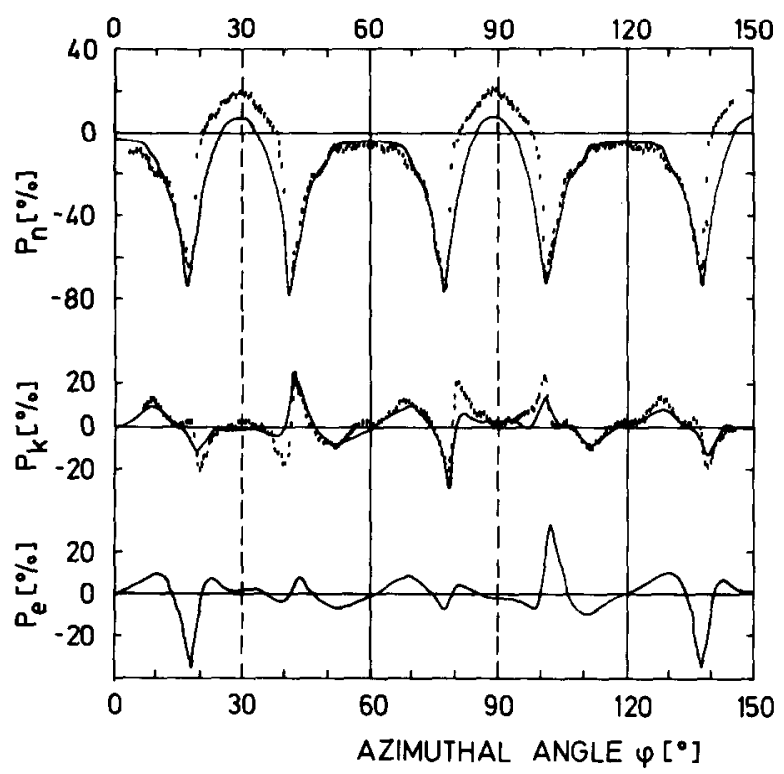

Fig. 2. Rotation diagrams from $\operatorname{Pt}(111)$ at $E=80 \mathrm{eV}$ and polar angle $\vartheta=47^{\circ}$ of the components $P_{n}$ (normal to the scattering plane), $P_{k}$ (longitudinal) and $P_{e}$ (in the scattering plane, or thogonal to directions $n$ and $k$ ) of the 00 beam spin polarization vector. Experiment (IIII) and theoretical results (-) calculated using ion-core potential $V_{E}, V_{\mathrm{i}}=4 \mathrm{eV}, T_{\mathrm{D}}=230 \mathrm{~K}$ and $\delta_{12}=0 \%$. 
the crystal, $P_{k}=0=P_{e}$, i.e. the polarization vector is normal to the scattering plane; (2) there is no mirror or antimirror symmetry with respect to $\varphi=30^{\circ}$ and $90^{\circ}$, and no six-fold rotation symmetry, which is induced for intensity rotation diagrams by time reversal invariance [34]. Spin polarization rotation diagrams thus do not have spatial symmetries beyond those of the crystal (cf. also ref. [2]). Since a monoatomic layer of $\mathrm{Pt}(111)$ has $\mathrm{C}_{6 \mathrm{v}}$ symmetry - as opposed to $\mathrm{C}_{3 \mathrm{v}}$ for two or more layers -, the finding of only $\mathrm{C}_{3 \mathrm{v}}$ symmetry is direct proof for the scattering contribution from subsurface layers. Since specular beam polarization rotation diagrams owe their existence to multiple scattering processes, the sharpness and size of the features in fig. 2 emphasize the importance of these processes.

The calculated $P_{n}$ and $P_{k}$ profiles in fig. 2 are seen to correspond very closely to their experimental counterparts with regard to existence, position and sign of peaks and - with the exception of $P_{n}$ around $30^{\circ}\left(\equiv 90^{\circ}\right)$ and $P_{k}$ near $38^{\circ}\left(\equiv 82^{\circ}\right)$ - even with regard to the absolute values. The sensitivity of the profiles is found to be selectively strong against changes in the ion-core potential model (cf. fig. $3 \mathrm{a}$ ). Comparison with experiment both for $P_{n}$ and $P_{k}$ immediately discards the band structure potential $V_{A}$ [18]. Discrimination between the band structure potential $V_{G}$ [19] and the potential $V_{E}$ with energy-dependent exchange is more subtle. It can be seen, however, from fig. 3a that both position and height of the negative $P_{n}$ peaks near $18^{\circ}$ and $42^{\circ}$ clearly favour $V_{E}$. Support for $V_{E}$ also comes from $P_{k}$ in the range between $40^{\circ}$ and $50^{\circ}$. Although $V_{G}$ improves the absolute height of the $P_{n}$ peak at $30^{\circ}$, we feel that this "pro" is outweighed by the "cons" just pointed out, and $V_{E}$ is superior to $V_{G}$. The effects of a reduction of the surface Debye temperature $T_{\mathrm{D}}^{\mathrm{s}}$, of changes in the imaginary potential $V_{\mathrm{i}}$ and of changes of the surface relaxation $\delta_{12}-$ shown in fig. $3 \mathrm{~b}$ for $P_{n}(\varphi)$ obtained from $V_{E}$ - are comparatively small. The reduction of $T_{\mathrm{D}}^{\mathrm{s}}$ from the bulk value 230 to $115 \mathrm{~K}$ is seen to produce about the same slight reduction of the negative peaks as does an increase of $V_{\mathrm{i}}$ from 4 to $5 \mathrm{eV}$. A similarity of the two effects is plausible, since the physical implication of both changes is an increase in absorption and thereby a reduction of multiple scattering. Comparison with experiment suggests to rule out $V_{\mathrm{i}}=5 \mathrm{eV}$ and the combinations $V_{\mathrm{i}}=4 \mathrm{eV}, T_{\mathrm{D}}^{\mathrm{s}}=230 \mathrm{~K}$ and $V_{\mathrm{i}}=3 \mathrm{eV}, T_{\mathrm{D}}^{\mathrm{s}}=115 \mathrm{~K}$. As will be shown later (fig. 8), there are, however, other profiles, which permit a discrimination. The insensitivity of $P_{n}(\varphi)$ to $\delta_{12}$ (fig. $3 \mathrm{~b}$, part C) does not even allow to discriminate between an inward and an outward relaxation by $4 \%$ of the bulk interlayer spacing, unless one overinterprets the small changes near $0^{\circ}$ and $60^{\circ}$. This finding, confirmed by other rotation diagrams (at $E=60 \mathrm{eV}$ ), seems particularly noteworthy in the light of a strong $\delta_{12}$ sensitivity obtained for some features in polarization rotation diagrams at $80 \mathrm{eV}$ from $\mathrm{W}(001)$ [8]. As a possible cause for this difference in $\delta_{12}$ sensitivity we suggest the relative weight of intra-layer and inter-layer multiple scattering processes in LEED from the two surfaces, the geometry of $\mathrm{W}(001)$ putting more weight on the inter-layer processes. Since the latter are responsible for effects of $\delta_{12}$ on $\boldsymbol{P}$, such effects should generally be stronger for W(001) than for Pt(111). 


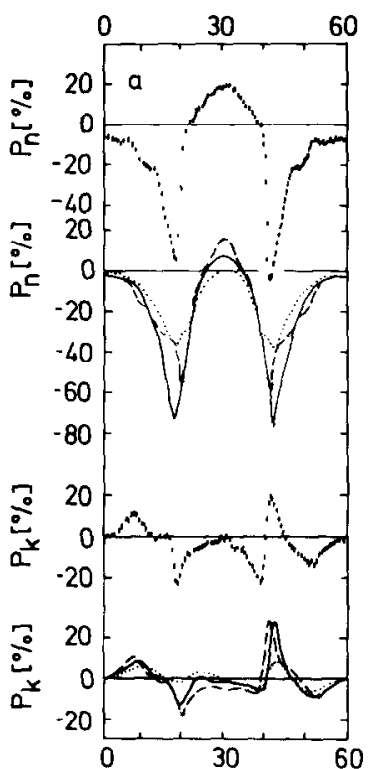

AZIMUTHAL ANGLE $\varphi\left[{ }^{\circ}\right]$

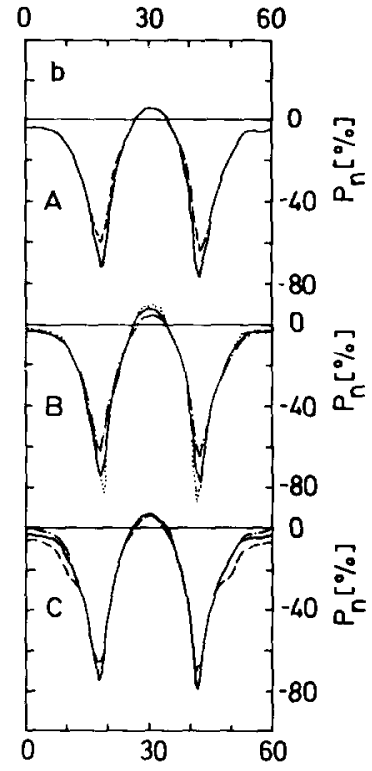

AZMMUTHAL ANGLE $\varphi\left[{ }^{\circ}\right]$

Fig. 3. Rotation diagrams at $E=80 \mathrm{eV}$ and $\vartheta=47^{\circ}$ of the 00 beam. (a) $P_{n}$ and $P_{k}$, experiment (IIII) and theory for ion-core potentials $V_{E}(\longrightarrow), V_{A}(\cdots \cdots)$ and $V_{G}(---), V_{\mathbf{i}}=4 \mathrm{eV}$, $T_{\mathrm{D}}=230 \mathrm{~K}$ and $\delta_{12}=0 \%$. (b) $P_{n}$, theory using $V_{E}$ : part A: $V_{1}=4 \mathrm{eV}, \delta_{12}=0 \%, T_{\mathrm{D}}=230 \mathrm{~K}$ $\left(\longrightarrow\right.$ ) and $115 \mathrm{~K}(---)$; part B: $T$ D $=230 \mathrm{~K}, \delta_{12}=0 \%, V_{\mathrm{i}}=3 \mathrm{eV}(\cdots \cdots), 4 \mathrm{eV}(\longrightarrow)$ and $5 \mathrm{eV}(-\cdot-\cdot-)$; part $\mathrm{C}: V_{\mathrm{i}}=4 \mathrm{eV}, T_{\mathrm{D}}^{\$}=230 \mathrm{~K}, \delta_{12}=-4 \%(-\cdot-\cdot-), 0 \%(-\longrightarrow)$ and $+4 \%(---)$.

For $\operatorname{Pt}(111)$, a strong $\delta_{12}$ sensitivity is found for nonspecular beam polarization versus polar angle $\vartheta$ profiles. This is illustrated by $P_{\overline{\mathbf{1}} \mathbf{0}}(\vartheta)$ in fig. 4 . Since the scatter ing plane coincides with a mirror plane of the crystal, the polarization vector is normal to this plane (cf. section 2.1), i.e. there is only $P_{n}$ to be considered. (The subscript $n$ can be replaced by the beam indices.) Focussing first on the results obtained for the "best" ion-core potential $V_{E}$, the following prominent changes are seen in progressing from an outward relaxation $\delta_{12}=4 \%$ to an inward relaxation $\delta_{12}=-4 \%$ : (1) the $P=+74 \%$ peak near $\vartheta=+21^{\circ}$ decreases to zero while slightly shifting to the right; (2) the $-8 \%$ shoulder near $\vartheta=+2^{\circ}$ grows into a $+66 \%$ peak; (3) the $+40 \%$ peak near $\vartheta=-14^{\circ}$ transforms, via a plus/minus feature with $-66 \%$, to a $+36 \%$ peak around $12^{\circ}$. The best fit to the experimental data (for $\vartheta>-10^{\circ}$ [35]) is seen to result for $0<\delta_{12}<+2 \%$. For the other two ion-core potentials $V_{A}$ and $V_{G}$, there is also a strong $\delta_{12}$ sensitivity. While $V_{A}$ is immediately seen to produce only poor fits for any $\delta_{12}$, the $V_{G}$ profile for $\delta_{12}=-2 \%$ might look reasonable at first glance. Closer inspection reveals, however, that the overall agreement with experiment is distinctly inferior to that obtained for $V_{E}$ and $\delta_{12}=0$ or 


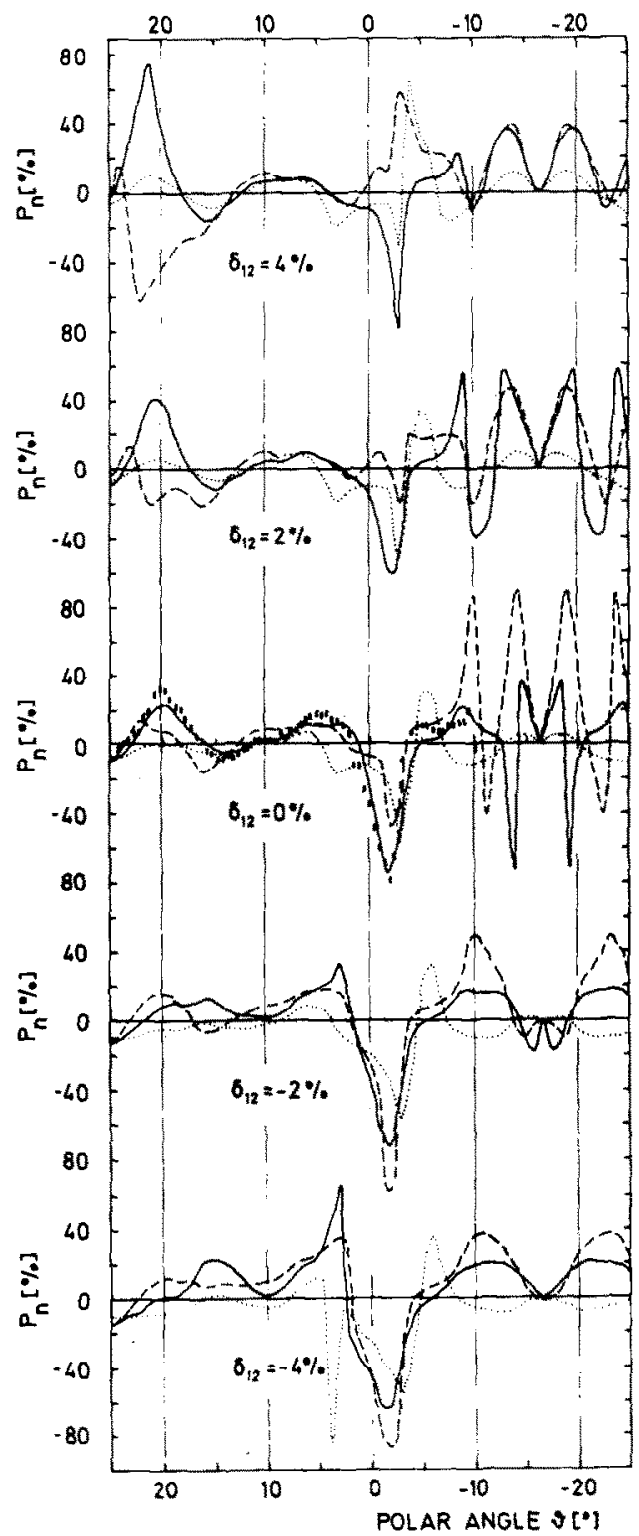

Fig. 4. Spin polarization (normal to the scattering plane) of the $\overline{10}$ beam versus angle $\vartheta$ for $E=80 \mathrm{eV}$ and scattering plane normal to the surface. Experiment (III) and theory using $V_{\mathrm{i}}=4 \mathrm{eV}, T \mathrm{G}=230 \mathrm{~K}$, surface relaxation as indicated and ion-core potentials $V_{E}(\longrightarrow$, $V_{A}(\cdots)$ and $V_{G}(-\cdots)$. 
$2 \%$. The influence of changes in the imaginary potential $V_{\mathrm{i}}$, of reducing the surface Debye temperature and of disregarding scattering by the surface barrier (no-reflection barrier) - shown in fig. 5 for $V_{E}$ and $\delta_{12}=0-$ is comparatively small for $\vartheta>-10^{\circ}$, i.e. in the range accessible within the present experimental set-up [35]. For $\vartheta<-10^{\circ}$, there is a strong sensitivity to $V_{\mathrm{i}}$ and $T_{\mathrm{D}}^{\mathrm{s}}$, which contains information on these quantities presently not retrievable due to the absence of experimental data.

For $\vartheta<-9^{\circ}$, the calculated profiles in figs. 4 and 5 are seen to reach zero at an angle $\vartheta_{\mathrm{s}}=-16.5^{\circ}$ and to be symmetrical with respect to $\vartheta_{\mathrm{s}}$. This is understood by noting that for $\vartheta=\vartheta_{\mathrm{s}}$ the direction of the diffracted $\overline{1} 0$ beam (polar angle $\vartheta^{\prime}$ ) is (anti)parallel to the direction of the incident beam, i.e. the scattering angle is $180^{\circ}$. For angles $\vartheta=\vartheta_{\mathrm{s}}+\epsilon$ we have, for small $\epsilon, \vartheta^{\prime}=\vartheta_{\mathrm{s}}-\epsilon$. Since the scattering plane coincides with a mirror plane of the crystal, time reversal symmetry (i.e. interchange of electron gun and detector) therefore dictates

$P_{\overline{1} 0}\left(\vartheta_{\mathrm{s}}+\epsilon\right)=-P_{\overline{1} 0}\left(\vartheta_{\mathrm{s}}-\epsilon\right)$,

where $P$ is defined with respect to a fixed direction (normal to the scattering plane) (cf. refs. $[13,14,34]$. In the present numerical results, $P$ is referred to $\boldsymbol{k}_{0} \times \boldsymbol{k} / k_{0} k$,

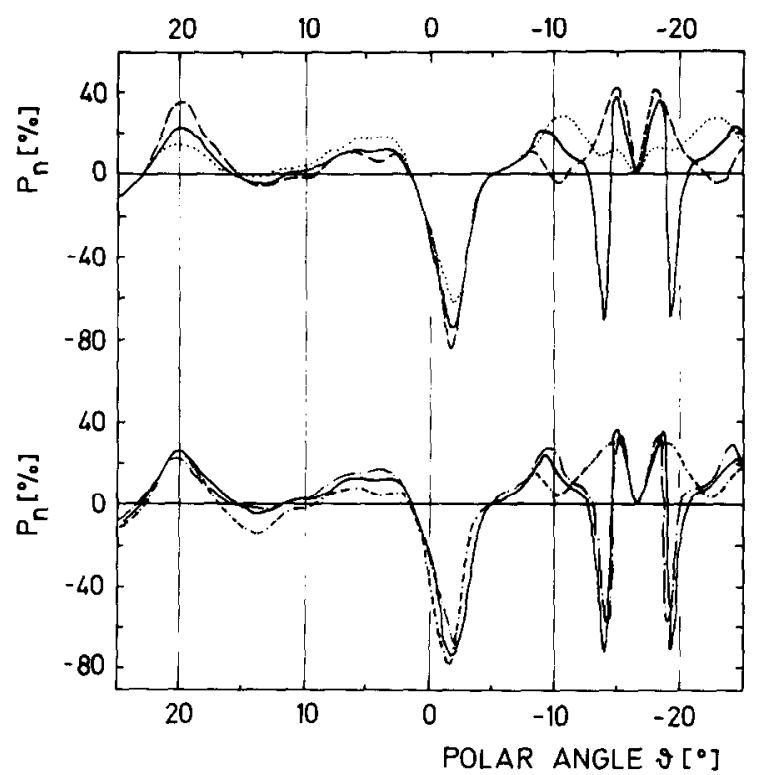

Fig. 5. Theoretical $P_{\overline{1} 0}(\vartheta)$ for diffraction conditions as in fig. 4, using ion-core potential $V_{E}$ and $\delta_{12}=0 \%$. Upper half: exponential-type surface barrier and $T_{\mathrm{D}}=230 \mathrm{~K}, V_{\mathrm{i}}=3 \mathrm{eV}$ $(---), 4 \mathrm{eV}(\longrightarrow)$ and $5 \mathrm{eV}(\cdots)$ ). Lower half: $V_{\mathrm{i}}=4 \mathrm{eV}$ and $T_{\mathrm{D}}^{\mathrm{S}}=230 \mathrm{~K}$ for exponential-type (-) and no-reflection surface barrier $(-\cdot-\cdot-) ; V_{\mathrm{i}}=4 \mathrm{eV}$ and exponential barrier for $T \mathrm{D}=115 \mathrm{~K}(--\cdot--)$. 
which changes sign as $\vartheta$ goes through $\vartheta_{s}$, and appears therefore symmetric instead of antisymmetric. In the limit $\epsilon \rightarrow 0$, the antisymmetry implies $P\left(\vartheta_{\mathrm{s}}\right)=0$.

The main results obtained from $P_{00}(\varphi)$ and $P_{\overline{1}_{0}}(\vartheta)$ at $E=80 \mathrm{eV}$, the preference for $V_{E}$ and $\delta_{12}$ between 0 and $+2 \%$, are supported by spin polarization versus profiles at other energy values. As an illustration, $P_{10}(\vartheta)$ at $95 \mathrm{eV}$ is shown in fig. 6 as obtained for $\delta_{12}=0$ and the three ion-core potentials. Again, $V_{E}$ is seen to yield the best agreement with experiment and $V_{A}$ the worst. Also, the sensitivity analysis to $\delta_{12}$, shown in fig. 7 for $V_{E}$, is found to favour $\delta_{12}=0$. Variations of $V_{\mathrm{i}}$ and $T_{\mathrm{D}}^{\mathrm{s}}$ produce only small changes. Like $P_{\overline{10}}(\vartheta)$ at $80 \mathrm{eV}$ (cf. fig. 5 ), $P_{10}(\vartheta)$ at $95 \mathrm{eV}$ shares with many other spin polarization profiles an insensitivity to the surface potential barrier model. The no-reflection barrier results being very similar, variations of the parameters of physically reasonable smooth barrier models have an even smaller effect. Some profiles, however, were found to exhibit barrier-sensitive features, which are associated with surface resonances (e.g. the $90 \mathrm{eV} P_{10}(\vartheta)$ profile shown in fig. 2 , cf. ref. [1]). Since these features also respond strongly to changes of $\delta_{12}$ and of the ion-core potential $V(r)$, they were not harnessed in the present analysis. They might, however, provide a stringent test for future ab initio surface potential models and for refinements of $V(r)$ beyond the presently "best" $V_{E}$.

While the polarization profiles, which have so far been discussed, were - at least in angular ranges studied experimentally - affected only mildly by reducing the surface Debye temperature $T_{\mathrm{D}}^{\mathrm{s}}$ and by varying the imaginary potential $V_{\mathrm{i}}$, we present in fig. 8 a more sensitive kind of profile. In the calculations, the previously established $\delta_{12}=0 \%$ and $V_{E}$ were used. The profiles obtained for $T_{\mathrm{D}}^{\mathrm{s}}=230 \mathrm{~K}$, i.e. the bulk value (see fig. $8 \mathrm{a}$ ), exhibit a striking change as $V_{\mathrm{i}}$ increases from 3 to $5 \mathrm{eV}$ : the sharp peaks near $22^{\circ}$ and $38^{\circ}$ are drastically reduced both in actual value and relative to the adjacent valleys. Agreement with experiment is seen to be best for

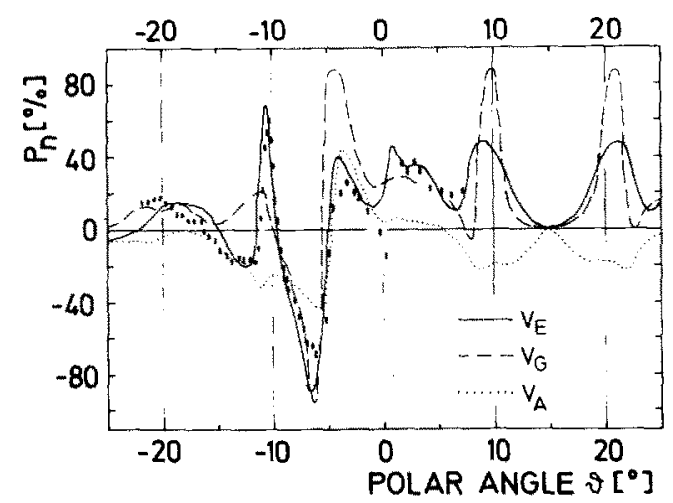

Fig. 6. Spin polarization (normal to the scattering plane of the 10 beam versus polar angle $\vartheta$ for $E=95 \mathrm{eV}$ and scattering plane normal to the surface. Experiment (III) and calculation for $\delta_{12}=0, V_{\mathrm{i}}=4 \mathrm{eV}, T_{\mathrm{D}}=230 \mathrm{~K}$ and the three ion-core potentials as indicated. 


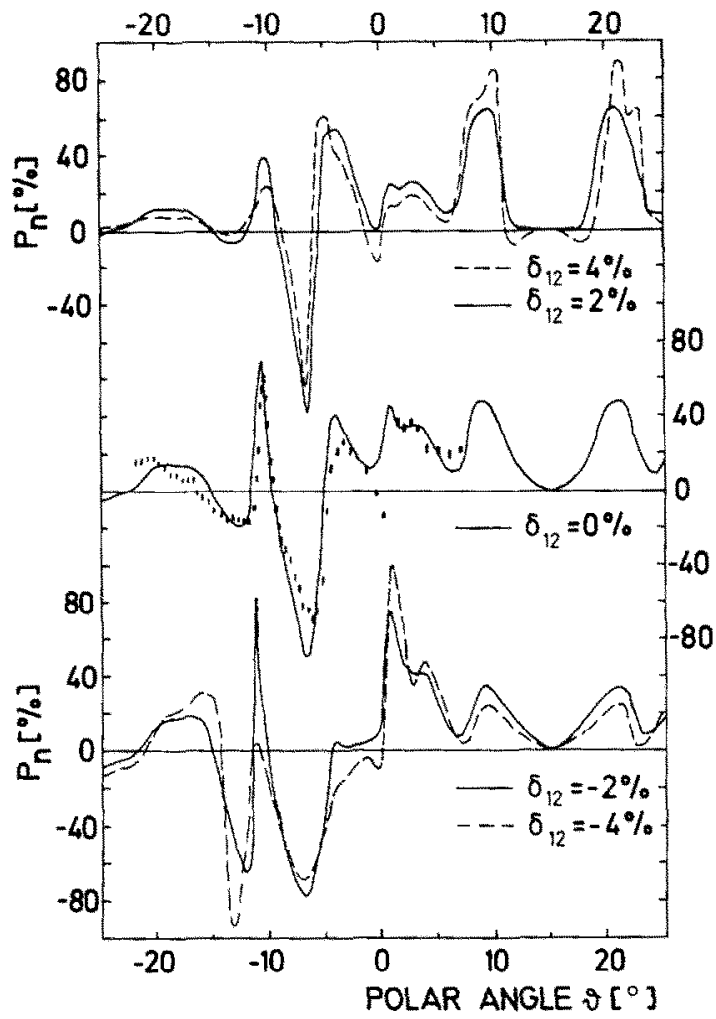

Fig. 7. $P_{10}(\vartheta)$ for diffraction conditions as in fig. 6. Experiment $(I I I)$ and calculation $(\longrightarrow)$ for $V_{\mathrm{i}}=4 \mathrm{eV}, T_{\mathrm{D}}=230 \mathrm{~K}$, ion-core potential $V_{E}$ and surface relaxation $\delta_{12}$ as indicated.

$V_{\mathrm{i}}$ between 3.5 and $4.0 \mathrm{cV}$; the values $V_{\mathrm{i}}=3$ and $5 \mathrm{eV}$ can be ruled out. Comparing the profiles obtained for $T_{\mathrm{D}}^{\mathrm{s}}=115 \mathrm{~K}$ (fig. $8 \mathrm{~b}$ ), we firstly note that, for $V_{\mathrm{i}}=4 \mathrm{eV}$, there is fairly little difference between using a Debye temperature of 115 and $230 \mathrm{~K}$ for the subsurface layers. Secondly, the effect of changing $V_{\mathrm{i}}$ is small by the standards of fig. $8 \mathrm{a}$. Thirdly, even the profile for $V_{\mathrm{i}}=3 \mathrm{eV}$ agrees rather poorly with experiment. We can thus not only rule out $V_{\mathrm{i}}=5 \mathrm{eV}$, as was anticipated from fig. $3 \mathrm{~b}$, but further discriminate between the two combinations $V_{\mathrm{i}}=4 \mathrm{eV}, T_{\mathrm{D}}^{\mathrm{s}}=230$ $\mathrm{K}$ and $V_{\mathrm{i}}=3 \mathrm{eV}, T_{\mathrm{D}}^{\mathrm{s}}=115 \mathrm{~K}$. The latter can be ruled out and a value of $T_{\mathrm{D}}^{\mathrm{s}}$ close to the bulk value $230 \mathrm{~K}$ seems likely. Since vibrational anisotropy has not been taken into account, this result can either mean that under the given diffraction conditions the parallel vibrations, which are described by a Debye temperature of about $230 \mathrm{~K}[25,28]$, dominate or that the perpendicular surface Debye temperature is not so far from the bulk value as in refs. [25] and [28].

The information gleaned from our $P(\vartheta)$ and $P(\varphi)$ profiles at constant energies is consistent with the message conveyed by the two non-specular beam profiles at 


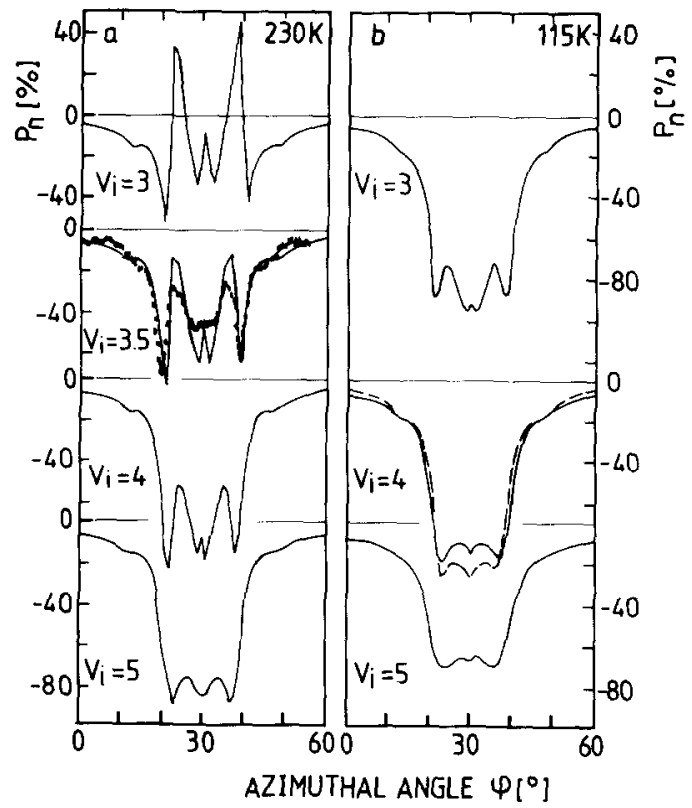

rig. 8. Specular beam $P_{n}$ rotation diagrams at $E=60 \mathrm{eV}$ and $\vartheta=43.5^{\circ}$. The calculations were all done for $V_{E}$ and $\delta_{12}=0 \%$. (a) Theoretical results ( $\longrightarrow$ ) for $T \mathrm{D}=230 \mathrm{~K}$ and imaginary potential $V_{\mathrm{i}}$ as indicated, and experiment (IIII). (b) Theoretical results for $V_{\mathrm{i}}$ as indicated, $T_{\mathrm{D}}^{\mathrm{S}}=115 \mathrm{~K}, T_{\mathrm{D}}=115 \mathrm{~K}(\longrightarrow$ ) and $230 \mathrm{~K}(---)$.

normal incidence, which we have studied as representatives of $P(E)$. However, this message is less precise. This may in part be due to the fact that our experimental data do not extend into the energy range above $110 \mathrm{cV}$, where calculations predict a higher sensitivity. On the other hand, it could also indicate a superiority of $P(\vartheta)$ and $P(\varphi)$ profiles over $P(E)$ profiles. As was pointed out in a recent LEED study using intensity rotation diagrams [36], a constant-energy mode offers several theoretical and experimental advantages.

\section{Conclusions}

The geometrical implication of the spin polarization profiles, which we have shown and discussed, is that the top layer of $\mathrm{Pt}(111)$ is almost unrelaxed. Taking into account the experimental uncertainties in energy, angles and spin polarization (cf. section 3), we arrive at a very slight outward relaxation $\delta_{12}=0.5 \% \pm 1 \%$ of the topmost interlayer spacing. This is in accordance with results from ion scattering $(1.5 \% \pm 1 \%[28]$ and $0.0 \% \pm 0.4 \%[17])$ and LEED intensity analyses $(0.0 \% \pm 2.5 \%$ 
[15] and $1.0 \% \pm 4.4 \%$ [16]). The agreement with the two LEED studies seems to indicate that the structural conclusion does not depend too crucially on the assumed ion-core potential, since these studies employed the band structure potential $V_{A}$, which the present work demonstrates to be distinctly inferior to a potential $\left(V_{E}\right)$ involving an energy-dependent exchange part [37].

As for the inner potential, its real part $V_{r}$ has been determined from our nonspecular $P(E)$ profiles at normal incidence as $12 \mathrm{eV}$ (cf. ref. [1]). This is close to the $14 \mathrm{eV}$ found in ref. [15] and far from the $5.42 \mathrm{eV}$ of ref. [16]. For the imaginary part $V_{\mathrm{i}}$ we find $3.5-4.0 \mathrm{cV}$ (in the range between 50 and $100 \mathrm{eV}$ ) in accordance with ref. [15]. The value $V_{\mathrm{i}}=5.18 \mathrm{eV}$ of ref. [16] is clearly too large below 100 $\mathrm{eV}$. As in ref. [15], use of the bulk Debye temperature $230 \mathrm{~K}$ for all layers appears adequate, although a reduced surface Debye temperature of about $200 \mathrm{~K}$ seems possible.

Our results demonstrate that spin polarization in LEED is a powerful means for surface analysis. The high sensitivity of numerous polarization features suggests that it might yield some information, e.g. on details of the scattering potential, with greater accuracy than intensity-only LEED. On the other hand, the larger computing time and storage requirements for polarization calculations would make a LEED surface structure determination via polarization-only too cumbersome. It seems therefore reasonable first to determine a model from intensities and then to confirm and possibly refine it with the aid of spin polarization. While the present LEED spin polarization study was done for a large- $Z$ surface $(Z=78)$, for which spin-orbit coupling is strong, recent results for $\mathrm{Ni}(001)(Z=28)$ suggest the usefulness of polarization also for low $Z Z$ materials [38].

\section{Acknowledgements}

Part of this work was done at the Institut für Festkörperforschung of the KFA Jülich, whose cooperation and hospitality we gratefully acknowledge. Also, we would like to thank Dr. D.W. Jepsen for making available his new matrix splitting routine, the use of which in our relativistic LEED programme made computations faster and more convenient, and Dr. W. Eckstein and Prof. Dr. D. Menzel for their continuous interest in and support of the experimental side of this work.

\section{References}

[1] P. Bauer, R. Feder and N. Müller, Surface Sci. 99 (1980) L395.

[2] P. Bauer, R. Feder and N. Müller, Solid State Commun. 36 (1980) 249.

[3] R. Feder, Phys. Status Solidi (b) 46 (1971) K31; 49 (1972) 699; 58 (1973) K137; 62 (1974) 135.

[4] R. Feder, Rev. Letters 36 (1976) 598.

[5] R. Feder, J. Phys. C14 (1981) 2049. 
[6] J.B. Pendry, Low Energy Electron Diffraction (Academic Press, London, 1974).

[7] M.A. Van Hove and S.Y. Tong, Surface Crystallography by LEED (Springer, Berlin, 1979).

[8] R. Feder and J. Kirschner, Surface Sci. 102 (1981), and references therein.

[9] P.J. Jennings, Surface Sci. 20 (1970) 18.

[10] R. Feder and J.B. Pendry, Solid State Commun, 26 (1978) 519.

[11] R. Feder, P.J. Jennings and R.O. Jones, Surface Sei. 61 (1976) 307.

[12] D.W. Jepsen, Phys. Rev. B22 (1980) 5701.

[13] R. Feder, Phys. Letters 78A (1980) 103.

[14] B.I. Dunlap, Solid State Commun. 35 (1980) 141.

[15] L.L. Kesmodel, P.C. Stair and G.G. Somorjai, Surface Sci. 64 (1977) 342.

[16] D.L. Adams, H.B. Nielsen and M.A. Van Hove, Phys. Rev. B20 (1979) 4789.

[17] J.A. Davies, D.P. Jackson, P.R. Norton, D.E. Posner and W.N. Unertl, Solid State Commun. 34 (1980) 41 .

[18] O.K. Andersen, Phys. Rev. B2 (1970) 883.

[19] D. Liberman, J.T. Waber and D.T. Cromer, Phys. Rev. 137 (1965) A27.

[20] J.S. Slater, Phys. Rev. 81 (1951) 385.

[21] D. Gloetzel, private communication (1977).

[22] L. Hedin and B.I. Lundquist, J. Phys. C4 (1971) 2064.

[23] J.C. Slater, T.M. Wilson and J.W. Wood, Phys. Rev. 179 (1969) 28.

[24] P.J. Bunyan and J.L. Schonfelder, Proc. Phys, Soc. 85 (1965) 455.

[25] H.B. Lyon and G.A. Somorjai, J. Chem. Phys, 44 (1966) 3707.

[26] D.P. Jackson, Surface Sci. 43 (1974) 431.

[27] E. B $\phi g h$ et al., in: Proc. 7th Intern. Vacuum Congr. and 3rd Intern. Conf. on Solid Surfaces, Vienna, 1977 , p. A-2707.

[28] F. van der Veen, R.G. Smeenk, R.M. Tromp and F.W. Saris, Surface Sci. 79 (1979) 219.

[29] The Debye temperature used in refs. [1] and [2] was actually $230 \mathrm{~K}$, while it was erroneously stated in ref. [1] as $178 \mathrm{~K}$.

[30] M. Ulehla and H.L. Davis, J. Vacuum Sci. Technol. 15 (1978) 642.

[31] R. Feder, N. Müller and D. Wolf, Z. Physik B28 (1977) 265.

[32] N. Müller, Dissertation, München (1977);

N. Müller, Report IPP 9/23 MPI für Plasmaphysik, Garching, 1979.

[33] The level of agreement between calculated and measured spin polarization profiles has been evaluated by visual inspection according to the criteria: (a) existence and position of peaks and "zero-crossings"; (b) sign and height of peaks; (c) general line shape. As regards the use of automated criteria of comparison ("agreement functionals" or " $R$-factors"), as have recently been employed in LEED intensity analyses (cf. J.B. Pendry, J. Phys, C13 (1980) 937, and references therein), we note that the formulae proposed for comparing intensity profiles are not necessarily appropriate for polarization profiles, since the latter are already normalized differences of intensity profiles.

[34] D.P. Woodruff and B.W. Holland, Phys. Letters 31A (1970) 207;

H.E. Farnsworth, Phys. Letters 36 A (1971) 56;

M.G. Lagally, T.C. Ngoc and M.B. Webb, Surface Sci. 25 (1971) 444.

[35] At $80 \mathrm{eV}, \vartheta=-10^{\circ}$ corresponds, for the 10 beam, to a scattering angle of $170^{\circ}$. For $\vartheta<10^{\circ}$, the beam is diffracted too close to the incident beam direction (electron gun) to be detectable.

[36] J. Kirschner and R. Feder, Surface Sci. 79 (1979) 176.

[37] LEED intensities obtained by using $V_{E}$ also agree better with measured intensities than do results from $V_{A}$ (see fig. 3 of: R. Feder, Surface Structure by LEED, Eds. P.M. Marcus and $F$. Jona, Plenum, in press).

[38] S.F. Alvarado, H. Hopster, R. Feder and H. Pleyer, Solid State Commun., in press. 